\title{
Visualizing Chemical Processes in Semiconductors with In Situ TEM
}

\section{Utkur Mirsaidov}

National University of Singapore, Singapore, Singapore

The continued demand for power-efficient and high-performance microelectronics components is the key driver for the increase in the density and miniaturization of transistors. ${ }^{1}$ The performance of these nanoscale transistors depends on their architecture and materials properties. ${ }^{2}$ Controlled fabrication of the nanoscale semiconductors materials tailored for microelectronics application requires detailed insight into the chemical and physical processes that take place during their fabrication. Optimization of these nanofabrication processes is currently based on trial-and-error approaches, and these structures are only characterized post-processing limiting the insight into how materials evolve during the fabrication.

Here, we combine advanced nanofabrication and in situ liquid cell TEM platform ${ }^{3}$ to create an advanced metrology problem to tackle key challenges in semiconductor manufacturing. Using our approach, we directly visualize the semiconductor fabrication related processes occurring in liquid chemicals with nanometer resolution in real-time. In my talk, I will show how we can study the etching of $\mathrm{Si}^{4}$ and $\mathrm{SiGe}^{5}$ nanowires and nanosheets, which will be an essential element in the next generation gate-all-around fieldeffect transistors (GAAFET). I will describe how the aspect-ratio of vertical nanostructures affects their failure modes during their fabrication. Furthermore, during my talk, I will show how crystallinity affects the etching at nanoscale and how the insights from the in situ studies enables to control the etch anisotropy to obtain different etch profiles, which are useful in fabrication of next generation transistors. Next, I will describe the application of in situ TEM to selective etching of semiconductors; I will show how in situ TEM helps us understand and devise the processes that enable to selectively etch one material, while leaving the other materials intact.

I will conclude by describing the potential future application of the in situ platform in microelectronics manufacturing.

Our results highlight the importance of visualizing the processes relevant to nanofabrication in order to understand and improve the nanofabrication related processes ${ }^{6}$.

\section{References}

[1] C. Thelander et al., Mater. Today 9 (2006), p.28.

[2] Waldrop, M., More Than Moore. Nature 530 (2016), p.145.

[3] U. Mirsaidov et al., Proc. Natl. Acad. Sci. U.S.A. 109 (2012), p.7187.

[4] Z. Aabdin et al., Nano Letters 17 (2017), p.2953.

[5] Zh. Baraissov et al., ACS Appl. Mater. Interfaces 11 (2019), p.36839.

[6] This work was supported by the Singapore National Research (NRF-CRP16-2015-05). 